\title{
Fine-tuning of gene expression dynamics by the Set2-Rpd3S pathway
}

\author{
Bo Bae Lee \\ Department of Life Science and the Research Center for Cellular Homeostasis, Ewha Womans University, Seoul 03760, Korea
}

\begin{abstract}
RNA polymerase II-interacting the Set2 methyltransferase co-transcriptionally methylates histone $\mathrm{H} 3$ at lysine 36 within the body of genes. This modification facilitates histone deacetylation by Rpd3S HDAC in $3^{\prime}$ transcribed regions to suppress cryptic initiation and slow elongation. Although this pathway is important for global deacetylation, no strong effects have been seen on genome-wide transcription under optimized laboratory conditions. In contrast, this pathway slows the kinetics of mRNA induction when target genes are induced upon environmental changes. Interestingly, a majority of Set2-repressed genes are overlapped by a IncRNA transcription that targets H3K36 methylation and deacetylation by Rpd3S HDAC to mRNA promoters. Furthermore, this pathway delays the induction of many cryptic transcripts upon environmental changes. Therefore, the Set2-Rpd3S HDAC pathway functions to fine-tune expression dynamics of mRNAs and ncRNAs. [BMB Reports 2017; 50(4): 162-163]
\end{abstract}

Chromatin, a complex of DNA and histones plays important roles in many processes including RNA polymerase II (RNA polII) transcription, DNA replication, and DNA repair. Nucleosome, the basic repeating unit of chromatin consists of four core histones, $(\mathrm{H} 3, \mathrm{H} 4, \mathrm{H} 2 \mathrm{~A}$, and $\mathrm{H} 2 \mathrm{~B})$ and 147 base pairs of DNA. Post-translational modifications of histones including acetylation, methylation, phosphorylation, and ubiquitination positively or negatively affect RNA polll transcription by regulating local nucleosome structure. Histone

*Corresponding author. E-mail: tskim@ewha.ac.kr

${ }^{\#}$ These authors contributed equally to this work.

https://doi.org/10.5483/BMBRep.2017.50.4.049

Received 17 March 2017

Keywords: Gene expression dynamics, H3K36 methylation, Histone deacetylation, Rpd3S HDAC, Set2

Abbreviations: HATs, histone acetyltransferases; HDACSs, histone deacetylases; RNA polII, RNA polymerase II; Rpd3S, Rpd3 small; lncRNA, long non-coding RNA

Perspective to: Kim et al. (2016) Nature Communications 7:13534, DOI: $10.1038 /$ ncomms13534 acetylation disrupts the interaction between DNA and histone proteins, and is highly correlated with transcription frequency. This modification is dynamically regulated by both histone acetyltransferases (HATs) and histone deacetylases (HDACs). Histone methylation also affects the local histone acetylation by acting as a binding site for either HATs or HDACs.

The Set2 methyltransferase preferentially interacts with phosphorylated CTD at both serine 5 and serine 2 of Rpb1, the largest subunit of RNA polll and methylates histone $\mathrm{H} 3$ at lysine 36 within the body of genes. H3K36 methylation by Set2 is involved in RNA polll elongation and co-transcriptional RNA processing. Since nucleosomes within the body of genes directly block elongation, histones need to be acetylated by HATs and evicted to promote elongation. However, once RNA polll gets transcribed through the gene, histone deacetylation is required to re-establish a repressive nucleosome structure and to suppress spurious transcription within the genes. The Set2-targeted H3K36 methylation facilitates histone deacetylation by Rpd3S(Rpd3 small) HDAC within coding regions. Two subunits, Eaf3 and Rco1, are important for binding of this complex to chromatin. Eaf3 chromodomain preferentially binds to methylated histones on H3K36 and Rco1 PHD finger interacts with unmodified histone tails. Both interactions stimulate histone deacetylation by Rpd3S HDAC. Although previous studies on the Set2-Rpd3S HDAC pathway have revealed the molecular mechanisms for targeting the pathway and inhibition of transcription from cryptic promoters, its function in mRNA transcription remains elusive. Interestingly, under optimized growth conditions, global gene expression pattern was not strongly affected in mutants for this pathway.

To further understand the function of the Set2-Rpd3S HDAC pathway in mRNA regulation, we analyzed genome-wide transcript levels in wild type and SET2 deleting cells during carbon source shifts. Although steady-state levels of transcripts were not changed, $\sim 80$ genes showed a more rapid induction in the absence of Set2. Furthermore, we also identified 757 cryptic promoters repressed by Set2. Interestingly, 416 cryptic promoters not reported previously were induced by distinct carbon sources. These findings suggest that upon environmental changes, the Set2-Rpd3S HDAC pathway has important roles in maintaining the optimal expression dynamics of mRNAs and non-coding RNAs (ncRNAs) (Fig. 1A).

Interestingly, a majority of the Set2-repressed genes had an 


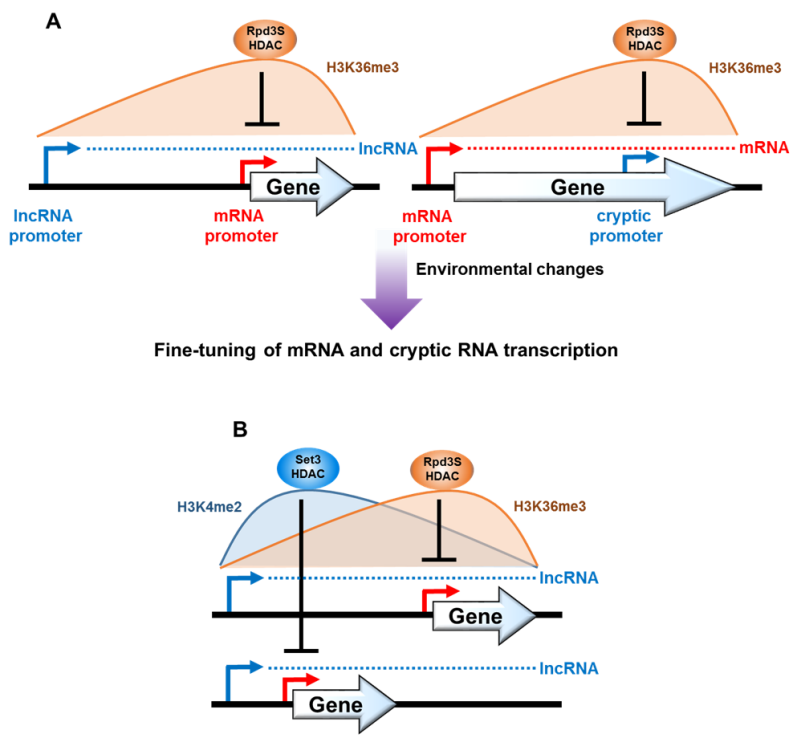

Fig. 1. Models for regulation of promoter dynamics by the Set2Rpd3S pathway. (A) IncRNA transcription from an upstream promoter targets H3K36me3 and Rpd3S HDAC to mRNA promoters. Histone deacetylation at mRNA promoters by Rpd3S HDAC delays the kinetics of gene induction upon environmental changes. Transcription from the mRNA promoter of a gene targets H3K36me3 and Rpd3S HDAC to inducible cryptic promoters. The resulting deacetylation by Rpd3S HDAC slows the rate of cryptic RNA induction. (B) Location of the IncRNA promoter determines the repressive effect of Set 3 HDAC and Rpd3S HDAC. H3K4me2 and H3K36me3 peak at $5^{\prime}$ and $3^{\prime}$ regions of IncRNA transcription, respectively. Set3 HDAC deacetylates H3K4me2-enriched nucleosomes to repress the mRNA promoter in $5^{\prime}$ regions. In $3^{\prime}$ regions of IncRNA transcription, Rpd3S HDAC deacetylates histones and represses mRNA promoters.

overlapping long non-coding RNA (IncRNA) transcription from an upstream or an antisense promoter. Furthermore, these genes showed non-canonical patterns of histone modifications. Although $\mathrm{H} 3 \mathrm{~K} 4 \mathrm{me} 3$ and $\mathrm{H} 3 \mathrm{~K} 36 \mathrm{me} 3$ are in general enriched at promoters and $3^{\prime}$ transcribed regions, respectively, Set2- repressed mRNA promoters showed high levels of H3K36me3 but not H3K4me3. We found that H3K36me3 at mRNA promoters targeted by an overlapping IncRNA transcription induced histone deacetylation by Rpd3S HDAC to delay the induction of target mRNA genes (Fig. 1A). A recent study from our group has revealed that H3K4me2-Set3 HDAC pathway also requires an overlapping IncRNA transcription to modulate the induction of target genes (Kim et al (2012) Cell 150, 1158-1169). Although the genes regulated by both H3K4me2-Set3 HDAC and Set2-Rpd3S HDAC pathways tend to be overlapped by IncRNA transcription, the two pathways affect the activation of mostly distinct target genes. An interesting difference between Set3- or Set2-repressed genes is the distance between mRNA and IncRNA promoters (Fig. 1B). Surprisingly, alteration of the distance between the mRNA and IncRNA promoters switches gene repression by the Set2-Rpd3S to H3K4me2-Set3 HDAC. Our findings suggest that although IncRNA can be transcribed from any site of a genome, locations of overlapping IncRNA promoters may be important for targeting distinct histone methylations and chromatin regulators to promoter regions of an associated mRNA gene (Fig. 1B).

Whereas gene-specific transcriptional activators or repressors primarily turn on or off their target genes, chromatin regulators appear to function as modulators that fine-tune the kinetics of gene induction or repression. This type of gene regulation seems to be important for cells growing in their natural conditions as they must reprogram gene expression patterns to adapt to rapidly changing environmental conditions. Therefore, fine-tuning of gene expression dynamics by chromatin regulators seems to be important for normal development processes, cellular adaptation, and proper responses to environmental stresses.

\section{ACKNOWLEDGEMENTS}

This work was supported by the National Research Foundation of Korea (NRF) grant funded by the Korea government (MSIP) (No. 2012R1A5A1048236). 